(migraine $48.5 \%$, tension-type $42.4 \%$ ). In the 3 months before the interview, $8.7 \%$ sought emergency services because of headaches, 30.8\% missed classes, 30.8\% were less productive, $75.6 \%$ used analgesics, $1.5 \%$ reported analgesic overuse, and headache had a substantial/severe impact on daily activities in $49 \%$. Multiple linear regressions showed that serious-impact headaches are significantly related to a greater number of subject failures and absenteeism, and are associated with worse academic performance. Neither anxiety (in $43.9 \%$ students) nor depression (in 18.9\%) had a significant effect on grade point average. No headache variables were associated with the grade point average coefficient, whereas individuals who consumed alcohol $(52.3 \%)$ had a smaller grade point average. (Souza-e-Silva HR, Roche-Filho PAS. Headaches and academic performance in university students: a cross-sectional study. Headache Nov-Dec 2011;51:1493-1502). (Respond: Dr Pedro AS Rocha-Filho, E-mail: pasrf@ig.com.br).

COMMENT. Headaches in 50\% of university students are severe and may be associated with poorer academic performance. Migraine prophylaxis and reduction of impact of headache severity on social and cognitive functioning might be expected to benefit academic performance. However, data from controlled studies of drugs frequently prescribed for migraine prophylaxis (amitryptiline, valproate, topiramate, and levetiracetam) are insufficient for appraisal. (Lewis D, et al. AAN Practice Parameter: pharmacological treatment of pediatric migraine headaches. Neurology 2004;63:22152224). Headache is not correlated with grade point average, whereas alcohol consumption has a significant association with a lower grade point average, and the risk of failure increases with the quantity of alcohol consumed. (Lopez-Frias M et al. J Stud Alcohol 2001;62:741-744, cited by authors).

\title{
COGNITIVE DISORDERS
}

\section{ROLE OF THE CEREBELLUM IN COGNITIVE FUNCTION}

Researchers in the Department of Psychology, University of Rome, Italy, retrospectively analyzed charts from patients in the Ataxia Lab of Santa Lucia Foundation between 1997 and 2007, focusing on the role of the cerebellum in cognition. Of 223 charts of cerebellar patients, mostly adults, 67 were excluded because the pathology was not restricted to the cerebellum; 156 comprising 84 males and 72 females were selected for analysis. Patients with focal or atrophic damage were grouped by etiology or location of the lesion. Focal lesions were ischemic or hemorrhagic stroke or surgical ablation for AV malformation or tumor. MRI was used to identify the lobular distribution of the lesion. Twelve different cerebellar atrophic lesions were represented by 16 cases of olivo-ponto-cerebellar atrophy, 15 idiopathic, 11 Friedreich's ataxia and the remainder as 1-5 cases each. In the clinical focal subgroup of 118 cases, 64 involved cerebellar deep nuclei, 25 the distribution of the posterior inferior cerebellar artery, and 12 the superior cerebellar artery.

Subjects with cerebellar damage had below average z-scores for all cognitive domains. Language, executive function, visuospatial abilities and sequencing are most severely affected functions. Subjects with lesions in the posterior inferior cerebellar artery territory exhibit the worst cognitive patterns, especially affecting sequencing, 
similar to those with lesions of the deep cerebellar nuclei. Vascular topography and involvement of deep cerebellar nuclei are the chief factors that determine the cerebellar cognitive profile. The findings support a model in which sequencing is the basic function of the cerebellum. (Tedesco AM, Chirocozzi FR, Clausi S, Lupo M, Molinari M. The cerebellar cognitive profile. Brain Dec 2011;134;3669-3683). (Respond: Marco Molinari MD PhD, Neurorehabilitation Santa Lucia Foundation, Via Ardeatina, 30600179 Roma, Italy. E-mail: m.molinari@hsantalucia.it).

COMMENT. The cerebellar cognitive affective syndrome includes impairments in executive functions, spatial cognition, language and personality changes in patients with cerebellar pathologies. In addition to executive function impairments, visuospatial functions, working memory, verbal memory, linguistic processing, verbal fluency, attention, sequencing and emotion are involved. This study including a large number of patients with cerebellar pathology provides a more comprehensive description of the variety and localization of cerebellar lesions and the specific type of cognition affected, especially sequencing. Subjects with lesions in the posterior inferior cerebellar artery territory and lesions in the deep cerebellar nuclei of the posterior lobe (dentate, emboliform, fastigial, globose nuclei) exhibit the worst cognitive patterns. Cerebellar lesions do not eliminate cognitive function, but they impair motor and mental task performance, causing a "dysmetria of thought." (Schmahmann JD and Sherman JC. The cerebellar cognitive affective syndrome. Brain 1998;121:561-579).

\section{LANGUAGE IMPAIRMENT AND ARACHNOID CYSTS}

Researchers at the Universite Catholique de Louvain, Brussels, Belgium, and Universite de Sherbrooke, Quebec, Canada studied 2 patients presenting with arachnoid cysts associated with cognitive impairment., particularly language impairment. Patient 1 , a 6-year-old, right-handed boy had a large head, articulation and phonology anomalies, and a normal intelligence. MRI revealed a left temporal lobe arachnoid cyst, and PET scan showed decreased metabolism in the left superior temporal gyrus and thalamus. A 24 hour-EEG was normal. A cysto-peritoneal shunt was placed at age 6 years. Postoperative MRI, 2 months after surgery, showed disappearance of the cyst and full reexpansion of the temporal lobe parenchyma. PET at 21 months postoperatively revealed a normal symmetric temporal lobe signal. The boy's phonology improved and was normal at 12 months postoperatively. Neuropsychological assessment repeated from 6 to 70 months postoperatively revealed language and attention span improvements, and an increase in full-scale IQ from 93 to 112, with no learning disabilities evident.

Patient 2, a 7-year-old, right-handed boy was admitted for chronic headaches of increasing intensity, associated with occasional vomiting. Neurologic exam was normal. Neuropsychological evaluation showed attention deficits, deficits in verbal comprehension, speech and syntactic, and in word definitions. Full scale IQ was normal. MRI at age 8 years showed a large arachnoid cyst at the base of the left sylvian fissure, causing a mass effect on the temporal lobe. 24 hour-EEG showed rare bursts of generalized spike and wave activity. A cysto-peritoneal shunt relieved the headaches and reduced the cyst volume. Postoperative EEG was similar to the original. Neuropsychological re-examination at 6 months showed improved verbal comprehension 